\title{
Stability Analysis for Markovian Jump Neutral Systems with Mixed Delays and Partially Known Transition Rates
}

\author{
Lianglin Xiong, ${ }^{1}$ Xiaobing Zhou, ${ }^{2}$ Jie Qiu, ${ }^{3}$ and Jing Lei ${ }^{1}$ \\ ${ }^{1}$ School of Mathematics and Computer Science, Yunnan University of Nationalities, \\ Kunming 650031, China \\ ${ }^{2}$ School of Information Science and Engineering, Yunnan University, Kunming 650091, China \\ ${ }^{3}$ Library of Yunnan, University of Nationalities, Kunming 650031, China \\ Correspondence should be addressed to Lianglin Xiong, lianglin_5318@126.com
}

Received 6 May 2012; Accepted 19 September 2012

Academic Editor: Ziemowit Popowicz

Copyright (C) 2012 Lianglin Xiong et al. This is an open access article distributed under the Creative Commons Attribution License, which permits unrestricted use, distribution, and reproduction in any medium, provided the original work is properly cited.

The delay-dependent stability problem is studied for Markovian jump neutral systems with partial information on transition probabilities, and the considered delays are mixed and model dependent. By constructing the new stochastic Lyapunov-Krasovskii functional, which combined the introduced free matrices with the analysis technique of matrix inequalities, a sufficient condition for the systems with fully known transition rates is firstly established. Then, making full use of the transition rate matrix, the results are obtained for the other case, and the uncertain neutral Markovian jump system with incomplete transition rates is also considered. Finally, to show the validity of the obtained results, three numerical examples are provided.

\section{Introduction}

A switched system is a dynamic system consisted of a number of subsystems and a rule that manages the switching between these subsystems. In the past, a large number of excellent papers and monographs on the stability of switched systems have been published such as [17] and the references cited therein. Among the results for switched systems, the stabilization problem of switched neutral systems has also been explored by some researchers [8-22], and mainly two kinds of switching rule are designed in these articles. Some state-dependent switching rules are obtained assuming the convex combination of the systems matrix, see, for example, $[8,10,20]$. To reduce the conservative, the authors in [11] have investigated the stabilization for switched neutral systems without the assumption that the restraint of 
the convex combination on systems matrices, the dwell time, and state-dependent rules were designed. Similarly, the authors in [12] have also studied the problem of the BIBO stability for switched neutral systems. Using the Razumikhin-like approach [13] and the LeibnizNewton formula, the global exponential stability for a class of switched neutral systems with interval-time-varying state delay and two classes of perturbations is investigated in [14], with arbitrary switching signal. Moreover, with the constructed state-dependent switching rule, the authors in [15] have investigated the global exponential stability of switched neutral systems. With the dwell time approach, the improved stability conditions for a class of switched neutral systems with mixed time-varying delays have also been obtained in [16], and the upper bound of derivative of the discrete time-varying delay is not restricted to one. In [17], the robust reliable stabilization of uncertain switched neutral systems with delayed switching has been considered. The $H \infty$ fault estimator for switched nonlinear systems of neutral type has been designed in [9]. In [18], the authors have studied the problem of exponential stability for neutral switched systems with interval time-varying mixed delays and nonlinear perturbations, obtaining the less conservative conditions based on the introduced free matrices. More recently, the markovian jump parameters have been considered for the analysis of switched neutral systems in [19].

In the past few decades, as a special switched system, markovian jump systems (MJSs) have been widely studied due to the fact that many dynamical systems subject to random abrupt variations can be modeled by MJSs such as manufacturing systems, networked control systems, and fault-tolerant control systems. There are a lot of useful results that have been presented in the literature, such as [23-29], and the references therein. For the analysis of MJSs, the transition probabilities in the jumping process determine the system behavior to a large extent. However, the likelihood of obtaining such available knowledge is actually questionable, and the cost is probably expensive. Rather than having a large complexity to measure or estimate all the transition probabilities, it is significant and necessary, from control perspectives, to further study more general jump systems with partly unknown transition probabilities. Recently, many results on the Markovian jump systems with partly unknown transition probabilities are obtained [30-37]. By introducing the free matrices based on the property of transition rate matrix, [32] gave less conservative conditions than that in [30] for Markovian jump systems with partial information on transition probability. And [33] provided with a new approach to obtain the necessary and sufficient conditions for markovian jump linear systems with incomplete transition probabilities, which may be appropriate to discuss the counterpart of delay systems. Most of these improved results just require some free matrices or the knowledge of the known elements in transition rate matrix, such as the bounds or structures of uncertainties, and some else of the unknown elements need not be considered. It is a great progress on the analysis of markovian jump systems. However, a few of these papers have considered the effect of delay on the stability or stabilization for the corresponding neutral systems, except for [19]. The global exponential stability of the markovian jumping neutral systems with interval time-varying delays has been studied by [19]; however, the transition probabilities are fully known, and the constructed Lyapunov did not fully consider the effect of the transition probabilities on the integrand. To the best of the authors' knowledge, the markovian jump neutral systems have not been fully investigated, and it is very challenging. All of these motivate this paper.

In this paper, the delay-dependent stability problem of neutral Markovian jump linear systems with partly unknown transition probabilities is investigated. The obtained results are presented in the form of linear matrix inequalities, which is easily computed by the Matlab toolbox. The considered systems are more general than the systems with completely 
known or completely unknown transition probabilities, which can be viewed as two special cases of the ones tackled here. Moreover, in contrast with the recent research on uncertain transition probabilities, our proposed concept of the partly unknown transition probabilities does not require any knowledge of the unknown elements, such as the bounds or structures of uncertainties. In addition, the relationship between the stability criteria currently obtained for the usual MJLS and switched linear system under arbitrary switching is exposed by our proposed systems. Furthermore, the number of matrix inequalities conditions obtained in this paper is much more than some existing results due to the introduced free matrices based on the system itself and the information of transition probabilities in this paper, which may increase the complexity of computation. However, it would decrease the conservativeness for the delay-dependent stability conditions. Finally, two numerical examples are provided to illustrate the effectiveness of our results.

\section{Problem Statement and Preliminaries}

Consider the following neutral system with markovian jump parameters:

$$
\begin{gathered}
\dot{x}(t)-C\left(r_{t}\right) \dot{x}\left(t-\tau_{2}\left(r_{t}\right)\right)=A\left(r_{t}\right) x(t)+B\left(r_{t}\right) x\left(t-\tau_{1}\left(r_{t}\right)\right), \\
x\left(t_{0}+\theta\right)=\varphi(\theta), \quad \forall \theta \in[-\tau, 0],
\end{gathered}
$$

where $\left\{r_{t}\right\}, t \geq 0$ is a right-continuous Markov process on the probability space taking values in a finite state space, $\wp=\{1,2, \ldots, N\}$ with generator $\mathbb{I}=\left(\lambda_{i j}\right), i, j \in \wp$ given by

$$
\operatorname{Pr}\left\{r_{t+\Delta}=j \mid r_{t}=i\right\}= \begin{cases}\lambda_{i j} \Delta+o(\Delta), & j \neq i, \\ 1+\lambda_{i i} \Delta+o(\Delta), & j=i,\end{cases}
$$

where $\Delta>0, \lim _{\Delta \rightarrow 0}(o(\Delta) / \Delta)=0, \lambda_{i j} \geq 0$, for $j \neq i$, is the transition rate from mode $i$ at time $t$ to mode $j$ at time $t+\Delta, \lambda_{i i}=-\sum_{j=1}^{N} \lambda_{i j} . A\left(r_{t}\right), B\left(r_{t}\right)$, and $C\left(r_{t}\right)$ are known matrix functions of the markovian process, $x(t) \in R^{n}$ is the state vector, and $\varphi(\theta)$ is the initial condition function. $\tau_{1}\left(r_{t}\right)$ and $\tau_{2}\left(r_{t}\right)$ are mode-dependent delays, when $r_{t}=i \in \wp, \tau_{1}\left(r_{t}\right)=\tau_{1 i}, \tau_{2}\left(r_{t}\right)=\tau_{2 i}$, and $\tau=\max \left(\tau_{1 i}, \tau_{2 i}\right)$.

Since the transition probability depends on the transition rates for the continuous-time MJSs, the transition rates of the jumping process are considered to be partly accessible in this paper. For instance, the transition rate matrix $\mathbb{I}$ with $\mathbb{N}$ operation modes may be expressed as

$$
\mathbb{I}=\left(\begin{array}{ccccc}
\lambda_{11} & ? & \lambda_{13} & \cdots & ? \\
? & ? & ? & ? & \lambda_{2 N} \\
\vdots & \vdots & \vdots & \ddots & \vdots \\
? & \lambda_{N 2} & \lambda_{N 3} & \cdots & \lambda_{N N}
\end{array}\right)
$$

where ? represents the unknown transition rate. For notational clarity, for all $i \in \wp$, the set $U^{i}$ denotes $U^{i}=U_{k}^{i} \cup U_{u k}^{i}$ with $U_{k}^{i} \triangleq\left\{j: \lambda_{i j}\right.$ is known for $\left.j \in \mathscr{Q}\right\}, U_{u k}^{i} \triangleq\left\{j: \lambda_{i j}\right.$ is unknown for $j \in \mathfrak{Q}\}$, and $\lambda_{k}^{i} \triangleq \sum_{j \in U_{k}^{i}} \lambda_{i j}$. 
Moreover, if $U_{k}^{i} \neq \emptyset$, it is further described as

$$
U_{k}^{i}=\left\{k_{1}^{i}, k_{2}^{i}, \ldots, k_{m}^{i}\right\}
$$

where $m$ is a nonnegation integer with $1 \leq m \leq N$, and $k_{j}^{i} \in Z^{+}, 1 \leq k_{j}^{i} \leq N j=1,2, \ldots, m$ represent the $j$ th known element of the set $U_{k}^{i}$ in the $i$ th row of the transition rate matrix $\mathbb{I}$.

Remark 2.1. It is worthwhile to note that if $U_{k}^{i}=\emptyset, U^{i}=U_{u k}^{i}$ which means that any information between the $i$ th mode and the other $N-1$ modes is not accessible, then MJSs with $N$ modes can be regarded as ones with $N-1$ modes. It is clear that when $U_{u k}^{i}=\emptyset, U^{i}=U_{k^{\prime}}^{i}$ the system (2.1) becomes the usual assumption case.

For the sake of simplicity, the solution $x\left(t, \varphi(\theta), r_{0}\right)$ with $r_{0} \in \wp$ is denoted by $x(t)$. It is known from [38] that $\{x(t), t\}$ is a Markov process with an initial state $\left\{\varphi(\theta), r_{0}\right\}$, and its weak infinitesimal generator, acting on function $V$, is defined in [39]:

$$
L V(x(t), t, i)=\lim _{\Delta \rightarrow 0^{+}} \frac{1}{\Delta}\left[\varepsilon\left(V\left(x(t+\Delta), t+\Delta, r_{t+\Delta}\right) \mid x(t), r_{t}=i\right)-V(x(t), t, i)\right]
$$

Throughout this paper, the following definition is necessary. More details refer to [23].

Definition 2.2 (see, [32]). The system (2.1) is said to be stochastically stable if the following holds:

$$
\varepsilon\left\{\int_{0}^{\infty}\|x(t)\|^{2} d t \mid \varphi, r_{0}\right\}<\infty
$$

for every initial condition $\varphi \in R^{n}$ and $r_{0} \in \wp$.

\section{Stability Analysis for Neutral Markovian Jump Systems}

The purpose of this section is to state the stability analysis for neutral markovian jump systems with partly unknown transition rates. Throughout the paper, the matrix $C\left(r_{t}\right)$ is assumed to be $\rho\left(C\left(r_{t}\right)\right)<1$. Before giving the stability result of systems (2.1) with a partly unknown transition rate matrix (2.3), the stability of neutral markovian jump systems (2.1) with all transition probabilities known is firstly investigated. With the introduced free matrices and the novel analysis technique of matrix, the stability conditions are presented in this section. 
Theorem 3.1. The system (2.1) with a fully known transition rate matrix is stochastically stable if there exist matrices $P_{i}>0, Q_{1 i}>0, Q_{2 i}>0, R_{1}>0$, and $R_{2}>0$ and any matrices $N_{1}, N_{2}, N_{3}$, and $N_{4}$ with appropriate dimensions satisfying the following linear matrix inequalities:

$$
\begin{gathered}
\varphi_{i}=\left(\begin{array}{cccc}
\varphi_{i 11} & \varphi_{i 12} & \varphi_{i 13} & \varphi_{i 14} \\
\star & \varphi_{i 22} & \varphi_{i 23} & \varphi_{i 24} \\
\star & \star & \varphi_{i 33} & \varphi_{i 34} \\
\star & \star & \star & \varphi_{i 44}
\end{array}\right)<0, \\
\sum_{j=1}^{N} \lambda_{i j} Q_{1 j}+\sum_{j=1}^{N} \lambda_{i j} \tau_{1 j} R_{1}-R_{1} \leq 0, \\
\sum_{j=1}^{N} \lambda_{i j} Q_{2 j}+\sum_{j=1}^{N} \lambda_{i j} \tau_{2 j} R_{2}-R_{2} \leq 0
\end{gathered}
$$

with

$$
\begin{gathered}
\varphi_{i 11}=P_{i} A_{i}+A_{i}^{T} P_{i}+\sum_{j=1}^{N} \lambda_{i j} P_{j}+Q_{1 i}+N_{1} A_{i}+A_{i}^{T} N_{1}^{T}+\tau_{1 i} R_{1}, \\
\varphi_{i 12}=-N_{1}+A_{i}^{T} N_{2}^{T}, \\
\varphi_{i 13}=N_{1} B_{i}+A_{i}^{T} N_{3}^{T}+P_{i} B_{i}, \\
\varphi_{i 14}=N_{1} C_{i}+A_{i}^{T} N_{4}^{T}+P_{i} C_{i}, \\
\varphi_{i 22}=-N_{2}-N_{2}^{T}+Q_{2 i}+\tau_{2 i} R_{2}, \\
\varphi_{i 23}=N_{2} B_{i}-N_{3}^{T}, \quad \varphi_{i 24}=N_{2} C_{i}-N_{4}^{T}, \\
\varphi_{i 33}=N_{3} B_{i}+B_{i}^{T} N_{3}^{T}-Q_{1 i}+\sum_{j=1}^{N} \lambda_{i j} \tau_{1 j} Q_{1 i}, \\
\varphi_{i 34}=N_{3} C_{i}+B_{i}^{T} N_{4}^{T}, \\
\varphi_{i 44}=N_{4} C_{i}+C_{i}^{T} N_{4}^{T}-Q_{2 i}+\sum_{j=1}^{N} \lambda_{i j} \tau_{2 j} Q_{2 i} .
\end{gathered}
$$

Proof. Construct a stochastic Lyapunov functional candidate as

$$
V\left(x_{t}, t, r_{t}\right)=\sum_{i=1}^{5} V_{i}\left(x_{t}, t, r_{t}\right)
$$


where

$$
\begin{gathered}
V_{1}\left(x_{t}, t, r_{t}\right)=x^{T}(t) P\left(r_{t}\right) x(t), \\
V_{2}\left(x_{t}, t, r_{t}\right)=\int_{t-\tau_{1}\left(r_{t}\right)}^{t} x^{T}(s) Q_{1}\left(r_{t}\right) x(s) d s, \\
V_{3}\left(x_{t}, t, r_{t}\right)=\int_{t-\tau_{2}\left(r_{t}\right)}^{t} \dot{x}^{T}(s) Q_{2}\left(r_{t}\right) \dot{x}(s) d s, \\
V_{4}\left(x_{t}, t, r_{t}\right)=\int_{-\tau_{1}\left(r_{t}\right)}^{0} \int_{t+\theta}^{t} x^{T}(s) R_{1} x(s) d s d \theta, \\
V_{5}\left(x_{t}, t, r_{t}\right)=\int_{-\tau_{2}\left(r_{t}\right)}^{0} \int_{t+\theta}^{t} \dot{x}^{T}(s) R_{2} \dot{x}(s) d s d \theta,
\end{gathered}
$$

where $P\left(r_{t}\right), Q_{1}\left(r_{t}\right), Q_{2}\left(r_{t}\right), R_{1}, R_{2}$, and $r_{t} \in \wp$ are all positive definite matrices with appropriate dimensions to be determined. Then, for given $r_{t}=i \in \wp, P\left(r_{t}\right)=P_{i}, Q_{1}\left(r_{t}\right)=$ $Q_{1 i}, Q_{2}\left(r_{t}\right)=Q_{2 i}$, and the weak infinitesimal operator $L$ of the stochastic process $x(t)$ along the evolution of $V_{k}\left(x_{t}, t, i\right)(k=1, \ldots, 7)$ are given as

$$
\begin{aligned}
L V_{1}\left(x_{t}, t, i\right) & =2 x^{T}(t) P_{i} \dot{x}(t)+x^{T}(t) \sum_{j=1}^{N} \lambda_{i j} P_{j} x(t) \\
& =2 x^{T}(t) P_{i}\left(A_{i} x(t)+B_{i} x\left(t-\tau_{1 i}\right)+C_{i} \dot{x}\left(t-\tau_{2 i}\right)\right)+x^{T}(t) \sum_{j=1}^{N} \lambda_{i j} P_{j} x(t) .
\end{aligned}
$$

According to the definition of the weak infinitesimal operator $L$ and the expression (2.2), it can be shown that

$$
\begin{aligned}
& L V_{2}\left(x_{t}, t, i\right) \\
& \left.\quad=\lim _{\Delta \rightarrow 0^{+}} \frac{1}{\Delta}\left[\varepsilon\left(\int_{t+\Delta-\tau_{1}\left(r_{t+\Delta}\right)}^{t+\Delta} x^{T}(s) Q_{1}\left(r_{t+\Delta}\right) x(s) d s\right) \mid x(t), r_{t}=i\right)-V_{2}(x(t), t, i)\right] \\
& =\lim _{\Delta \rightarrow 0^{+}} \frac{1}{\Delta}\left[\int_{t+\Delta-\tau_{1}\left(r_{t+\Delta}\right)}^{t+\Delta} x^{T}(s) \sum_{j=1}^{N} \operatorname{Pr}\left\{\mathrm{r}_{\mathrm{t}+\Delta}=\mathrm{j} \mid \mathrm{r}_{\mathrm{t}}=\mathrm{i}\right\} \mathrm{Q}_{1 \mathrm{j}} \mathrm{x}(\mathrm{s}) \mathrm{ds}-\mathrm{V}_{2}(\mathrm{x}(\mathrm{t}), \mathrm{t}, \mathrm{i})\right] \\
& =\lim _{\Delta \rightarrow 0^{+}} \frac{1}{\Delta}\left[\int_{t+\Delta-\tau_{1 i}-\sum_{j=1}^{N}\left(\lambda_{i j} \Delta+O(\Delta)\right) \tau_{1 j}}^{t+\Delta} x^{T}(s)\left[Q_{1 i}+\sum_{j=1}^{N}\left(\lambda_{i j} \Delta+O(\Delta)\right) Q_{1 j}\right] x(\mathrm{~s}) d s\right. \\
& =\lim _{\Delta \rightarrow 0^{+}} \frac{1}{\Delta}\left[\int_{t+\Delta-\tau_{1 i}-\sum_{j=1}^{N}\left(\lambda_{i j} \Delta+O(\Delta)\right) \tau_{1 j}}^{t+\int_{t-\tau_{1 i}} x^{T}(s) Q_{1 i} x(s) d s}\right]
\end{aligned}
$$


Abstract and Applied Analysis

$$
\begin{aligned}
& \quad+\lim _{\Delta \rightarrow 0^{+}} \frac{1}{\Delta}\left[\int_{t+\Delta-\tau_{1 i}-\sum_{j=1}^{N}\left(\lambda_{i j} \Delta+O(\Delta)\right) \tau_{1 j}}^{t+\Delta} x^{T}(s)\left(\sum_{j=1}^{N}\left(\lambda_{i j} \Delta+O(\Delta)\right) Q_{1 j}\right) x(s) d s\right] \\
& =\lim _{\Delta \rightarrow 0^{+}} \frac{1}{\Delta} \int_{t}^{t+\Delta} x^{T}(s) Q_{1 i} x(s) d s \\
& \quad+\lim _{\Delta \rightarrow 0^{+}} \frac{1}{\Delta} \int_{t+\Delta-\tau_{1 i}-\sum_{j=1}^{N}\left(\lambda_{i j} \Delta+O(\Delta)\right) \tau_{1 j}}^{t-\tau_{1 i}} x^{T}(s) Q_{1 i} x(s) d s \\
& +\lim _{\Delta \rightarrow 0^{+}} \frac{1}{\Delta} \int_{t+\Delta-\tau_{1 i}-\sum_{j=1}^{N}\left(\lambda_{i j} \Delta+O(\Delta)\right) \tau_{1 j}}^{t-\tau_{1 i}} x^{T}(s) \sum_{j=1}^{N}\left(\lambda_{i j} \Delta+O(\Delta)\right) Q_{1 j} x(s) d s \\
& =x^{T}(t) Q_{1 i} x(t)-\left(1-\sum_{j=1}^{N} \lambda_{i j} \tau_{1 j}\right) x^{T}\left(t-\tau_{1 i}\right) Q_{1 i} x\left(t-\tau_{1 i}\right) \\
& \quad+\int_{t-\tau_{1 i}}^{t} x^{T}(s) \sum_{j=1}^{N} \lambda_{i j} Q_{1 j} x(s) d s,
\end{aligned}
$$

$L V_{4}\left(x_{t}, t, i\right)$

$$
\begin{aligned}
\left.=\lim _{\Delta \rightarrow 0^{+}} \frac{1}{\Delta}\left[\varepsilon\left(\int_{-\tau_{1}\left(r_{t+\Delta}\right)}^{0} \int_{t+\Delta+\theta}^{t+\Delta} x^{T}(s) R_{1} x(s) d s\right) \mid x(t), r_{t}=i\right)-\int_{-\tau_{1 i}}^{0} \int_{t+\theta}^{t} x^{T}(s) R_{1} x(s) d s\right] \\
=\lim _{\Delta \rightarrow 0^{+}} \frac{1}{\Delta}\left[\varepsilon\left(\int_{-\tau_{1 i}-\sum_{j=1}^{N}\left(\lambda_{i j} \Delta+O(\Delta)\right) \tau_{1 j}}^{0} \int_{t+\Delta+\theta}^{t+\Delta} x^{T}(s) R_{1} x(s) d s\right) \mid x(t), r_{t}=i\right) \\
\left.\quad-\int_{-\tau_{1 i}}^{0} \int_{t+\theta}^{t} x^{T}(s) R_{1} x(s) d s\right] \\
=\lim _{\Delta \rightarrow 0^{+}} \frac{1}{\Delta}\left[\varepsilon\left(\int_{-\tau_{1 i}-\sum_{j=1}^{N}\left(\lambda_{i j} \Delta+O(\Delta)\right) \tau_{1 j}}^{-\tau_{i}} \int_{t+\Delta+\theta}^{t+\Delta} x^{T}(s) R_{1} x(s) d s\right) \mid x(t), r_{t}=i\right) \\
\left.\quad+\int_{-\tau_{1 i}}^{0} \int_{t+\Delta+\theta}^{t+\Delta} x^{T}(s) R_{1} x(s) d s-\int_{-\tau_{1 i}}^{0} \int_{t+\theta}^{t} x^{T}(s) R_{1} x(s) d s\right] \\
=\tau_{1 i} x^{T}(t) R_{1} x(t)-\int_{t-\tau_{1 i}}^{t} x^{T}(s) R_{1} x(s) d s+\int_{t-\tau_{1 i}}^{t} x^{T}(s) \sum_{j=1}^{N} \lambda_{i j} \tau_{1 j} R_{1} x(s) d s .
\end{aligned}
$$

Similar to the above, we can obtain

$$
L V_{3}\left(x_{t}, t, i\right)=\dot{x}^{T}(t) Q_{2 i} \dot{x}(t)-\left(1-\sum_{j=1}^{N} \lambda_{i j} \tau_{2 j}\right) \dot{x}^{T}\left(t-\tau_{2 i}\right) Q_{2 i} \dot{x}\left(t-\tau_{2 i}\right)
$$




$$
\begin{gathered}
+\int_{t-\tau_{2 i}}^{t} \dot{x}^{T}(s) \sum_{j=1}^{N} \lambda_{i j} Q_{2 j} \dot{x}(s) d s, \\
L V_{5}\left(x_{t}, t, i\right)=\tau_{2 i} \dot{x}^{T}(t) R_{2} \dot{x}(t)-\int_{t-\tau_{2 i}}^{t} \dot{x}^{T}(s) R_{2} \dot{x}(s) d s \\
+\int_{t-\tau_{2 i}}^{t} \dot{x}^{T}(s) \sum_{j=1}^{N} \lambda_{i j} \tau_{2 j} R_{2} \dot{x}(s) d s .
\end{gathered}
$$

Moreover, there exist matrices $N_{k}(k=1, \ldots, 4)$ with appropriate dimensions, such that the following equality holds according to (2.1):

$$
2 \xi^{T}(t) N\left(-\dot{x}(t)+A_{i} x(t)+B_{i} x\left(t-\tau_{1 i}\right)+C_{i} \dot{x}\left(t-\tau_{2 i}\right)\right)=0,
$$

where

$$
\begin{gathered}
N^{T}=\left(\begin{array}{llll}
N_{1}^{T} & N_{2}^{T} & N_{3}^{T} & N_{4}^{T}
\end{array}\right) \\
\xi^{T}(t)=\left(\begin{array}{llll}
x^{T}(t) & \dot{x}^{T}(t) & x^{T}\left(t-\tau_{1 i}\right) & \dot{x}^{T}\left(t-\tau_{2 i}\right)
\end{array}\right) .
\end{gathered}
$$

From (3.7)-(3.10) and with (3.2)-(3.3), one can obtain that

$$
L V\left(x_{t}, t, i\right)=\sum_{j=1}^{4} L V_{j}\left(x_{t}, t, i\right)=x^{T}(t) \varphi_{i} x(t)<0
$$

where $\varphi_{i}$ are defined in this theorem. Therefore,

$$
\varepsilon\left\{\int_{0}^{\infty}\|x(t)\|^{2} d t \mid \varphi, r_{0}\right\}<\infty
$$

which means that systems (2.1) are stochastic stability. The proof is completed.

Based on the result of Theorem 3.1, the next theorem will relate to the stability condition of systems (2.1) with partially known transition probabilities.

Theorem 3.2. The system (2.1) with a partly unknown transition rate matrix (2.4) is stochastically stable if there exist matrices $P_{i}>0, Q_{1 i}>0, Q_{2 i}>0(i=1,2, \ldots, N), R_{1}>0$, and $R_{2}>0$ and 
any matrices $N_{k}(k=1, \ldots, 4), W_{i}=W_{i}^{T}$ with appropriate dimensions satisfying the following linear matrix inequalities:

$$
\begin{aligned}
& \phi_{i}=\left(\begin{array}{cccc}
\phi_{i 11} & \phi_{i 12} & \phi_{i 13} & \phi_{i 14} \\
\star & \phi_{i 22} & \phi_{i 23} & \phi_{i 24} \\
\star & \star & \phi_{i 33} & \phi_{i 34} \\
\star & \star & \star & \phi_{i 44}
\end{array}\right)<0, \quad \forall j \in U_{u k}^{i} \text { if } i \in U_{k^{\prime}}^{i} \\
& \psi_{i}=\left(\begin{array}{cccc}
\psi_{i 11} & \psi_{i 12} & \psi_{i 13} & \psi_{i 14} \\
\star & \psi_{i 22} & \psi_{i 23} & \psi_{i 24} \\
\star & \star & \psi_{i 33} & \psi_{i 34} \\
\star & \star & \star & \psi_{i 44}
\end{array}\right)<0, \quad \forall j \in U_{u k}^{i} \text { if } i \in U_{u k^{\prime}}^{i} \\
& \sum_{j \in U_{k}^{i}} \lambda_{i j} Q_{1 j}+\sum_{j \in U_{k}^{i}} \lambda_{i j} \tau_{1 j} R_{1}-R_{1}-\lambda_{k}^{i} Q_{1 j}-\lambda_{k}^{i} \tau_{1 j} R_{1} \leq 0, \quad \forall j \in U_{u k}^{i} \quad \text { if } i \in U_{k^{\prime}}^{i} \\
& \sum_{j \in U_{k}^{i}} \lambda_{i j} Q_{1 j}+\sum_{j \in U_{k}^{i}} \lambda_{i j} \tau_{1 j} R_{1}-R_{1}+\lambda_{d}^{i} Q_{1 i}-\lambda_{d}^{i} Q_{1 j}-\lambda_{k}^{i} Q_{1 j}+\lambda_{d}^{i} \tau_{1 i} R_{1} \\
& -\lambda_{d}^{i} \tau_{1 j} R_{1}-\lambda_{k}^{i} \tau_{1 j} R_{1} \leq 0, \quad \forall j \in U_{u k}^{i} \text { if } i \in U_{k^{\prime}}^{i} \\
& \sum_{j \in U_{k}^{i}} \lambda_{i j} Q_{2 j}+\sum_{j \in U_{k}^{i}} \lambda_{i j} \tau_{2 j} R_{2}-R_{2}-\lambda_{k}^{i} Q_{2 j}-\lambda_{k}^{i} \tau_{2 j} R_{2} \leq 0, \quad \forall j \in U_{u k}^{i} \quad \text { if } i \in U_{k^{\prime}}^{i} \\
& \sum_{j \in U_{k}^{i}} \lambda_{i j} Q_{2 j}+\sum_{j \in U_{k}^{i}} \lambda_{i j} \tau_{2 j} R_{2}-R_{2}+\lambda_{d}^{i} Q_{2 i}-\lambda_{d}^{i} Q_{2 j}-\lambda_{k}^{i} Q_{2 j}+\lambda_{d}^{i} \tau_{2 i} R_{2} \\
& -\lambda_{d}^{i} \tau_{2 j} R_{2}-\lambda_{k}^{i} \tau_{2 j} R_{2} \leq 0, \quad \forall j \in U_{u k}^{i} \quad \text { if } i \in U_{k}^{i} \\
& P_{j}-W_{i} \leq 0, \quad j \in U_{u k^{\prime}}^{i} j \neq i, \\
& P_{j}-W_{i} \geq 0, \quad j \in U_{u k^{\prime}}^{i} j=i,
\end{aligned}
$$

with

$$
\begin{gathered}
\phi_{i 11}=P_{i} A_{i}+A_{i}^{T} P_{i}+\sum_{j \in U_{k}^{i}} \lambda_{i j}\left(P_{j}-W_{i}\right)+Q_{1 i}+N_{1} A_{i}+A_{i}^{T} N_{1}^{T}+\tau_{1 i} R_{1}, \\
\phi_{i 12}=-N_{1}+A_{i}^{T} N_{2}^{T}, \\
\phi_{i 13}=N_{1} B_{i}+A_{i}^{T} N_{3}^{T}+P_{i} B_{i},
\end{gathered}
$$




$$
\begin{aligned}
& \phi_{i 14}=N_{1} C_{i}+A_{i}^{T} N_{4}^{T}+P_{i} C_{i}, \\
& \phi_{i 22}=-N_{2}-N_{2}^{T}+Q_{2 i}+\tau_{2 i} R_{2} \\
& \phi_{i 23}=N_{2} B_{i}-N_{3}^{T} \text {, } \\
& \phi_{i 24}=N_{2} C_{i}-N_{4}^{T}, \\
& \phi_{i 33}=N_{3} B_{i}+B_{i}^{T} N_{3}^{T}-Q_{1 i}+\sum_{j \in U_{k}^{i}} \lambda_{i j} \tau_{1 j} Q_{1 i}-\lambda_{k}^{i} \tau_{1 j} Q_{1 i} \\
& \phi_{i 34}=N_{3} C_{i}+B_{i}^{T} N_{4}^{T} \text {, } \\
& \phi_{i 44}=N_{4} C_{i}+C_{i}^{T} N_{4}^{T}-Q_{2 i}+\sum_{j \in U_{k}^{i}} \lambda_{i j} \tau_{2 j} Q_{2 i}-\lambda_{k}^{i} \tau_{2 j} Q_{2 i} . \\
& \psi_{i 33}=N_{3} B_{i}+B_{i}^{T} N_{3}^{T}-Q_{1 i}+\sum_{j \in U_{k}^{i}} \lambda_{i j} \tau_{1 j} Q_{1 i}+\lambda_{d}^{i} \tau_{1 i} Q_{1 i}-\lambda_{d}^{i} \tau_{1 j} Q_{1 i}-\lambda_{k}^{i} \tau_{1 j} Q_{1 i}, \\
& \psi_{i 44}=N_{4} C_{i}+C_{i}^{T} N_{4}^{T}-Q_{2 i}+\sum_{j \in U_{k}^{i}} \lambda_{i j} \tau_{2 j} Q_{2 i}+\lambda_{d}^{i} \tau_{2 i} Q_{2 i}-\lambda_{d}^{i} \tau_{2 j} Q_{2 i}-\lambda_{k}^{i} \tau_{2 j} Q_{2 i}, \\
& \psi_{i 11}=\phi_{i 11}, \quad \psi_{i 12}=\phi_{i 12}, \quad \psi_{i 13}=\phi_{i 13}, \quad \psi_{i 14}=\phi_{i 14}, \\
& \psi_{i 22}=\phi_{i 22}, \quad \psi_{i 23}=\phi_{i 23}, \quad \psi_{i 24}=\phi_{i 24}, \quad \psi_{i 34}=\phi_{i 34},
\end{aligned}
$$

and $\lambda_{d}^{i}$ is a given lower bound for the unknown diagonal element.

Proof. For the case of the systems (2.1) with partly unknown transition probabilities, and taking into account the situation that the information of transition probabilities is not accessible completely, due to $\sum_{j=1}^{N} \lambda_{i j}=0$, the following zero equation holds for arbitrary matrices $W_{i}=W_{i}^{T}$ is satisfied:

$$
-x^{T}(t)\left(\sum_{j=1}^{N} \lambda_{i j} W_{i}\right) x(t)=0, \quad \forall i \in \mathfrak{\wp},
$$

and the inequality (3.12) can be rewritten as

$$
L V\left(x_{t}, t, i\right)=x^{T}(t) \sum_{j \in U_{u k}^{i}} \lambda_{i j}\left(P_{j}-W_{i}\right) x(t)+\xi^{T}(t) \tilde{\varphi}_{i} \xi(t)<0,
$$

where $\xi(t)$ has already been defined on the above

$$
\tilde{\varphi}_{i}=\left(\begin{array}{cccc}
\tilde{\varphi}_{i 11} & \tilde{\varphi}_{i 12} & \tilde{\varphi}_{i 13} & \tilde{\varphi}_{i 14} \\
\star & \widetilde{\varphi}_{i 22} & \widetilde{\varphi}_{i 23} & \tilde{\varphi}_{i 24} \\
\star & \star & \widetilde{\varphi}_{i 33} & \widetilde{\varphi}_{i 34} \\
\star & \star & \star & \tilde{\varphi}_{i 44}
\end{array}\right), \quad i=1, \ldots, N,
$$


with the elements are the same as those in $\varphi_{i}$, except for

$$
\tilde{\varphi}_{i 11}=P_{i} A_{i}+A_{i}^{T} P_{i}+\sum_{j \in U_{k}^{i}} \lambda_{i j}\left(P_{j}-W_{i}\right)+Q_{1 i}+N_{1} A_{i}+A_{i}^{T} N_{1}^{T}
$$

and note that $\lambda_{i i}=-\sum_{j \in U_{k}^{i}} \lambda_{i j}$ and $\lambda_{i j} \geq 0$ for all $j \neq i$, namely, $\lambda_{i i}<0$ for all $i \in \wp$. Therefore, it follows from easy computation that if $i \in U_{k^{\prime}}^{i}$,inequalities (3.20) and the formula (3.25) less than 0 imply that

$$
L V\left(x_{t}, t, i\right)<0 \text {. }
$$

On the other hand, for the same reason, if $i \in U_{u k}^{i}$, inequalities (3.20)-(3.21) and the formula (3.25) less than 0 also imply that inequality (3.27) holds. Therefore,

$$
\varepsilon\left\{\int_{0}^{\infty}\|x(t)\|^{2} d t \mid \varphi, r_{0}\right\}<\infty
$$

which means that systems (2.1) with partly unknown transition probabilities are stochastically stable.

Note that the formula (3.25) less than 0 can be represented as follows:

$$
\tilde{\varphi}_{i}=\Phi_{i}+\sum_{j \in U_{u k}^{i}} \bar{\lambda}_{i j} \operatorname{diag}\left(0,0, \tau_{1 j} Q_{1 i}, \tau_{2 j} Q_{2 i}\right)<0
$$

where $\bar{\lambda}_{i j}$ is an unknown element in transition probabilities matrix, and

$$
\Phi_{i}=\left(\begin{array}{cccc}
\Phi_{i 11} & \Phi_{i 12} & \Phi_{i 13} & \Phi_{i 14} \\
\star & \Phi_{i 22} & \Phi_{i 23} & \Phi_{i 24} \\
\star & \star & \Phi_{i 33} & \Phi_{i 34} \\
\star & \star & \star & \Phi_{i 44}
\end{array}\right)
$$

with

$$
\begin{gathered}
\Phi_{i 11}=\tilde{\varphi}_{i 11}, \quad \Phi_{i 12}=\tilde{\varphi}_{i 12}, \quad \Phi_{i 13}=\tilde{\varphi}_{i 13}, \quad \Phi_{i 14}=\tilde{\varphi}_{i 14}, \\
\Phi_{i 22}=\tilde{\varphi}_{i 22}, \quad \Phi_{i 23}=\tilde{\varphi}_{i 23}, \quad \Phi_{i 24}=\tilde{\varphi}_{i 24}, \quad \Phi_{i 34}=\tilde{\varphi}_{i 34}, \\
\Phi_{i 33}=N_{3} B_{i}+B_{i}^{T} N_{3}^{T}-Q_{1 i}+\sum_{j \in U_{k}^{i}} \lambda_{i j} \tau_{1 j} Q_{1 i}, \\
\Phi_{i 44}=N_{4} C_{i}+C_{i}^{T} N_{4}^{T}-Q_{2 i}+\sum_{j \in U_{k}^{i}} \lambda_{i j} \tau_{2 j} Q_{2 i} .
\end{gathered}
$$

One can note that (3.29) can be separated into two cases, $i \in U_{k}^{i}$ and $i \in U_{u k}^{i}$. 
Case I $\left(i \in U_{k}^{i}\right)$ : it should be first noted that in this case one has $\lambda_{k}^{i}<0$. In fact, we only need to consider $\lambda_{k}^{i}<0$ because $\lambda_{k}^{i}=0$ means all the elements in the $i$ throw of the transition rate matrix.

Now the formula (3.29) can be rewritten as

$$
\tilde{\varphi}_{i}=\Phi_{i}+\sum_{j \in U_{u k}^{i}} \bar{\lambda}_{i j} \operatorname{diag}\left(0,0, \tau_{1 j} Q_{1 i}, \tau_{2 j} Q_{2 i}\right)=\Phi_{i}-\lambda_{k}^{i} \sum_{j \in U_{u k}^{i}} \frac{\bar{\lambda}_{i j}}{-\lambda_{k}^{i}} \operatorname{diag}\left(0,0, \tau_{1 j} Q_{1 i}, \tau_{2 j} Q_{2 i}\right)<0
$$

It follows from $0 \leq \bar{\lambda}_{i j} /-\lambda_{k}^{i} \leq 1$ and $\sum_{j \in U_{u k}^{i}}\left(\bar{\lambda}_{i j} /-\lambda_{k}^{i}\right)=1$ that

$$
\tilde{\varphi}_{i}=\sum_{j \in U_{u k}^{i}} \frac{\bar{\lambda}_{i j}}{-\lambda_{k}^{i}}\left(\Phi_{i}-\lambda_{k}^{i} \operatorname{diag}\left(0,0, \tau_{1 j} Q_{1 i}, \tau_{2 j} Q_{2 i}\right)\right)<0
$$

Similar to the above proof, (3.2) and (3.3) can be rewritten as (3.16) and (3.18), respectively, for this case. Accordingly, for $0 \leq \bar{\lambda}_{i j} \leq \lambda_{k}^{i}, \tilde{\varphi}_{i}<0$ is equivalent to (3.14) which is satisfied for all $j \in U_{u k}^{i}$, which also implies that, in the presence of unknown elements $\bar{\lambda}_{i j}$, the system stability is ensured if (3.14), (3.16), (3.18), and (3.20) hold.

Case II $\left(i \in U_{u k}^{i}\right)$ : for the sake of simple expression, let $\Psi_{j}=\operatorname{diag}\left(0,0, \tau_{1 j} Q_{1 i}, \tau_{2 j} Q_{2 i}\right)$.

In this case, $\bar{\lambda}_{i i}$ is unknown, $\lambda_{k}^{i} \geq 0$, and $\bar{\lambda}_{i i} \leq 0$, and following the same analysis of the above case, we just consider $\bar{\lambda}_{i i}<-\lambda_{k}^{i}$. And now the formula (3.29) can be rewritten as

$$
\begin{aligned}
\tilde{\varphi}_{i} & =\Phi_{i}+\bar{\lambda}_{i i} \operatorname{diag}\left(0,0, \tau_{1 i} Q_{1}, \tau_{2 i} Q_{2}\right)+\sum_{j \in U_{u k}^{i}, j \neq i} \bar{\lambda}_{i j} \operatorname{diag}\left(0,0, \tau_{1 j} Q_{1 i}, \tau_{2 j} Q_{2 i}\right) \\
& =\Phi_{i}+\bar{\lambda}_{i i} \operatorname{diag}\left(0,0, \tau_{1 i} Q_{1 i}, \tau_{2 i} Q_{2 i}\right)+\left(-\bar{\lambda}_{i i}-\lambda_{k}^{i}\right) \sum_{j \in U_{u k}^{i}, j \neq i} \frac{\bar{\lambda}_{i j}}{-\bar{\lambda}_{i i}-\lambda_{k}^{i}} \Psi_{j} .
\end{aligned}
$$

Similarly, since we have

$$
0 \leq \frac{\bar{\lambda}_{i j}}{-\bar{\lambda}_{i i}-\lambda_{k}^{i}} \leq 1, \quad \sum_{j \in U_{u k^{i}}^{i} j \neq i} \frac{\bar{\lambda}_{i j}}{-\bar{\lambda}_{i i}-\lambda_{k}^{i}}=1,
$$

one can know that

$$
\tilde{\varphi}_{i}=\sum_{j \in U_{u k}^{i}} \frac{\bar{\lambda}_{i j}}{-\bar{\lambda}_{i i}-\lambda_{k}^{i}}\left(\Phi_{i}+\bar{\lambda}_{i i} \Psi_{i}+\left(-\bar{\lambda}_{i i}-\lambda_{k}^{i}\right) \Psi_{j}\right)
$$

which means that $\tilde{\varphi}_{i}<0$ is equivalent to all $j \in U_{u k^{\prime}}^{i} j \neq i$,

$$
\Phi_{i}+\bar{\lambda}_{i i} \Psi_{i}+\left(-\bar{\lambda}_{i i}-\lambda_{k}^{i}\right) \Psi_{j}<0
$$


and from the defined $\lambda_{d}^{i}$ in Theorem 3.2, we have that $\lambda_{d}^{i} \leq \bar{\lambda}_{i i}<-\lambda_{k^{\prime}}^{i}$ which means that $\bar{\lambda}_{i i}$ may take any value between $\left[\lambda_{d}^{i},-\lambda_{k}^{i}+\varepsilon\right]$ for some $\varepsilon<0$ arbitrarily small. Then, $\bar{\lambda}_{i i}$ can be further written as a convex combination

$$
\bar{\lambda}_{i i}=\alpha\left(-\lambda_{k}^{i}+\varepsilon\right)+(1-\alpha) \lambda_{d^{\prime}}^{i}
$$

where $\alpha$ takes value arbitrarily in [0,1]. So, (3.37) holds if and only if for all $j \in U_{u k^{\prime}}^{i}, j \neq i$,

$$
\begin{gathered}
\Phi_{i}-\lambda_{k}^{i} \Psi_{i}+\varepsilon\left(\Psi_{i}-\Psi_{j}\right)<0 \\
\Phi_{i}+\lambda_{d}^{i} \Psi_{i}-\lambda_{d}^{i} \Psi_{j}-\lambda_{k}^{i} \Psi_{i}<0
\end{gathered}
$$

simultaneously hold. Since $\varepsilon$ is arbitrarily small, (3.39) holds if and only if

$$
\Phi_{i}-\lambda_{k}^{i} \Psi_{i}<0
$$

which is the case in (3.40) when $j=i$ for all $j \in U_{u k}^{i}$. Hence, (3.37) is equivalent to (3.15). Furthermore, following the same line of this proof, (3.2) and (3.3) can be represented as (3.17) and (3.19), respectively, in this case.

Therefore, from the above discussion, in the presence of unknown elements in the transition probabilities matrix, we can readily draw a conclusion that the system (2.1) with partly known transition rates is stable if the inequalities in Theorem 3.2 are satisfied. It completes this proof.

Remark 3.3. In order to obtain the less conservative stability criterion of MJSs with partial information on transition probabilities, similar to [32], the free-connection weighting matrices are introduced by making use of the relationship of the transition rates among various subsystems, that is, $\sum_{j=1}^{N} \lambda_{i j}=0$ for all $i \in \wp$, which overcomes the conservativeness of using the fixed-connection weighting matrices. However, it is difficult to decrease the conservative using free-connection matrices only based on the above equalities, but not on the systems and the themselves Newton-Leibniz formula. Moreover, this paper is inspired by [30], and the delay-dependent stability results in this theorem are the extension of [30] to delay systems to some extent. Although the large number of introduced free weighting matrices may increase the complexity of computation, using the technique of free weighting matrices would reduce the conservativeness, which would be reflected in the fifth section.

Remark 3.4. It should be noted that the more known elements are there in (2.3), the lower the conservative of the condition will be. In other word, the more unknown elements are there in (2.3), the lower the maximum of time delay will be in Theorem 3.2. Actually, if all transition probabilities are unknown, the corresponding system can be viewed as a switched linear system under arbitrary switching. Thus, the conditions obtained in Theorem 3.2 will thereby cover the results for arbitrary switched linear system with mixed delays. In that case, one can see that the stability condition in Theorem 3.2 becomes seriously conservative, for many constraints. Fortunately, we can use the common Lyapunov functional method to analyze the stability for the system under the assumption that all transition probabilities are not known. 
For the stability analysis of the neutral markovian jump systems with all transition probabilities is not known, one can construct the following common Lyapunov functional:

$$
\begin{aligned}
V\left(x_{t}, t, r_{t}\right)= & x^{T}(t) P x(t)+\int_{t-\tau_{1}\left(r_{t}\right)}^{t} x^{T}(s) Q_{1} x(s) d s+\int_{t-\tau_{2}\left(r_{t}\right)}^{t} \dot{x}^{T}(s) Q_{2} \dot{x}(s) d s \\
& +\int_{-\tau_{1}\left(r_{t}\right)}^{0} \int_{t+\theta}^{t} x^{T}(s) R_{1} x(s) d s d \theta+\int_{-\tau_{2}\left(r_{t}\right)}^{0} \int_{t+\theta}^{t} \dot{x}^{T}(s) R_{2} \dot{x}(s) d s d \theta,
\end{aligned}
$$

and following a similar line as in the proof of Theorem 3.1, we can obtain the following corollary.

Corollary 3.5. The system (2.1) with all elements unknown in transition rate matrix (2.3) is stochastically stable if there exist positive definite matrices $P, Q_{1}, Q_{2}, R_{1}>0$, and $R_{2}>0$ and any matrices $N_{k}(k=1, \ldots, 4)$ with appropriate dimensions satisfying the following linear matrix inequalities:

$$
x_{i}=\left(\begin{array}{cccc}
x_{i 11} & x_{i 12} & x_{i 13} & x_{i 14} \\
\star & x_{i 22} & x_{i 23} & x_{i 24} \\
\star & \star & x_{i 33} & x_{i 34} \\
\star & \star & \star & x_{i 44}
\end{array}\right)<0
$$

with

$$
\begin{gathered}
X_{i 11}=P A_{i}+A_{i}^{T} P+Q_{1}+N_{1} A_{i}+A_{i}^{T} N_{1}^{T}+\tau_{1 i} R_{1}, \\
\chi_{i 12}=-N_{1}+A_{i}^{T} N_{2}^{T}, \\
x_{i 13}=N_{1} B_{i}+A_{i}^{T} N_{3}^{T}+P B_{i}, \\
x_{i 14}=N_{1} C_{i}+A_{i}^{T} N_{4}^{T}+P C_{i}, \\
x_{i 22}=-N_{2}-N_{2}^{T}+Q_{2}+\tau_{2 i} R_{2}, \\
x_{i 23}=N_{2} B_{i}-N_{3}^{T}, \\
x_{i 24}=N_{2} C_{i}-N_{4}^{T}, \\
x_{i 33}=N_{3} B_{i}+B_{i}^{T} N_{3}^{T}-Q_{1}, \\
x_{i 34}=N_{3} C_{i}+B_{i}^{T} N_{4}^{T}, \\
x_{i 44}=N_{4} C_{i}+C_{i}^{T} N_{4}^{T}-Q_{2} .
\end{gathered}
$$




\section{Extension to Uncertain Neutral Markov Jump Systems}

In this section, we will consider the uncertain neutral Markov jump systems with partially unknown transition probabilities as follows:

$$
\dot{x}(t)-C\left(r_{t}\right) \dot{x}\left(t-\tau_{2}\left(r_{t}\right)\right)=\left(A\left(r_{t}\right)+\Delta A\left(r_{t}\right)\right) x(t)+\left(B\left(r_{t}\right)+\Delta B\left(r_{t}\right)\right) x\left(t-\tau_{1}\left(r_{t}\right)\right) .
$$

$A\left(r_{t}\right), B\left(r_{t}\right)$ are known mode-dependent constant matrices with appropriate dimensions, while $\Delta A\left(r_{t}\right), \Delta B\left(r_{t}\right)$ are the time-varying but norm-bounded uncertainties satisfying

$$
\left(\Delta A\left(r_{t}\right) \Delta B\left(r_{t}\right)\right)=L_{r_{t}} F_{r_{t}}(t)\left(H_{1}\left(r_{t}\right) H_{2}\left(r_{t}\right)\right)
$$

where $L_{r_{t}}, H_{1}\left(r_{t}\right)$, and $H_{2}\left(r_{t}\right)$ are known mode-dependent matrices with appropriate dimensions, and $F_{r_{t}}(t)$ is the time-varying unknown matrix function with Lebesgue norm measurable elements satisfying $F_{r_{t}}^{T}(t) F_{r_{t}}(t) \leq I$.

Theorem 4.1. The uncertain neutral markovian jump system (4.1) with a partly unknown transition rate matrix (2.3) is stochastically stable if there exist matrices $\widetilde{P}_{i}>0, \widetilde{Q}_{1 i}>0, \widetilde{Q}_{2 i}>0, \widetilde{R}_{1}>0$, and $\widetilde{R}_{2}>0$ and any matrices $\widetilde{W}_{i}=\widetilde{W}_{i}^{T}, \widetilde{M}_{i}=\widetilde{M}_{i}^{T}, \widetilde{S}_{i}=\widetilde{S}_{i}^{T}, \widetilde{F}_{k}, \widetilde{G}_{k}$, and $\widetilde{N}_{k}(k=1, \ldots, 6)$ with appropriate dimensions satisfying the following linear matrix inequalities:

$$
\begin{aligned}
& \tilde{\phi}_{i}=\left(\begin{array}{cccccc}
\tilde{\phi}_{i 11} & \tilde{\phi}_{i 12} & \tilde{\phi}_{i 13} & \tilde{\phi}_{i 14} & H_{1 i}^{T} & \widetilde{P}_{i} L_{i}+\widetilde{N}_{1} L_{i} \\
\star & \widetilde{\phi}_{i 22} & \widetilde{\phi}_{i 23} & \widetilde{\phi}_{i 24} & 0 & \widetilde{N}_{2} L_{i} \\
\star & \star & \widetilde{\phi}_{i 33} & \widetilde{\phi}_{i 34} & H_{2 i}^{T} & \widetilde{N}_{3} L_{i} \\
\star & \star & \star & \widetilde{\phi}_{i 44} & 0 & \widetilde{N}_{4} L_{i} \\
\star & \star & \star & \star & -I & 0 \\
\star & \star & \star & \star & \star & -I
\end{array}\right)<0, \quad \forall j \in U_{u k}^{i} \quad \text { if } i \in U_{k^{\prime}}^{i} \\
& \widetilde{\psi}_{i}=\left(\begin{array}{cccccc}
\widetilde{\psi}_{i 11} & \widetilde{\varphi}_{i 12} & \widetilde{\psi}_{i 13} & \widetilde{\psi}_{i 14} & H_{1 i}^{T} & \widetilde{P}_{i} L_{i}+\widetilde{N}_{1} L_{i} \\
\star & \widetilde{\psi}_{i 22} & \widetilde{\psi}_{i 23} & \widetilde{\psi}_{i 24} & 0 & \widetilde{N}_{2} L_{i} \\
\star & \star & \widetilde{\varphi}_{i 33} & \widetilde{\varphi}_{i 34} & H_{2 i}^{T} & \widetilde{N}_{3} L_{i} \\
\star & \star & \star & \widetilde{\psi}_{i 44} & 0 & \widetilde{N}_{4} L_{i} \\
\star & \star & \star & \star & -I & 0 \\
\star & \star & \star & \star & \star & -I
\end{array}\right)<0, \quad \forall j \in U_{u k}^{i} \quad \text { if } i \in U_{u k}^{i}
\end{aligned}
$$




$$
\begin{aligned}
& \sum_{j \in U_{k}^{i}} \lambda_{i j} \widetilde{Q}_{1 j}+\sum_{j \in U_{k}^{i}} \lambda_{i j} \tau_{1 j} \widetilde{R}_{1}-\widetilde{R}_{1}-\lambda_{k}^{i} \widetilde{Q}_{1 j}-\lambda_{k}^{i} \tau_{1 j} \widetilde{R}_{1} \leq 0, \forall j \in U_{u k}^{i} \text { if } i \in U_{k^{\prime}}^{i} \\
& \sum_{j \in U_{k}^{i}} \lambda_{i j} \widetilde{Q}_{1 j}+\sum_{j \in U_{k}^{i}} \lambda_{i j} \tau_{1 j} \widetilde{R}_{1}-\widetilde{R}_{1}+\lambda_{d}^{i} \widetilde{Q}_{1 i}-\lambda_{d}^{i} \widetilde{Q}_{1 j}-\lambda_{k}^{i} \widetilde{Q}_{1 j} \\
& +\lambda_{d}^{i} \tau_{1 i} \widetilde{R}_{1}-\lambda_{d}^{i} \tau_{1 j} \widetilde{R}_{1}-\lambda_{k}^{i} \tau_{1 j} \widetilde{R}_{1} \leq 0, \quad \forall j \in U_{u k}^{i} \quad \text { if } i \in U_{k}^{i}, \\
& \sum_{j \in U_{k}^{i}} \lambda_{i j} \widetilde{Q}_{2 j}+\sum_{j \in U_{k}^{i}} \lambda_{i j} \tau_{2 j} \widetilde{R}_{2}-\widetilde{R}_{2}-\lambda_{k}^{i} \widetilde{Q}_{2 j}-\lambda_{k}^{i} \tau_{2 j} \widetilde{R}_{2} \leq 0, \quad \forall j \in U_{u k}^{i} \text { if } i \in U_{k^{\prime}}^{i} \\
& \sum_{j \in U_{k}^{i}} \lambda_{i j} \widetilde{Q}_{2 j}+\sum_{j \in U_{k}^{i}} \lambda_{i j} \tau_{2 j} \widetilde{R}_{2}-\widetilde{R}_{2}+\lambda_{d}^{i} \widetilde{Q}_{2 i}-\lambda_{d}^{i} \widetilde{Q}_{2 j}-\lambda_{k}^{i} \widetilde{Q}_{2 j} \\
& +\lambda_{d}^{i} \tau_{2 i} \widetilde{R}_{2}-\lambda_{d}^{i} \tau_{2 j} \widetilde{R}_{2}-\lambda_{k}^{i} \tau_{2 j} \widetilde{R}_{2} \leq 0, \quad \forall j \in U_{u k}^{i} \quad \text { if } i \in U_{k}^{i} \\
& \widetilde{P}_{j}-\widetilde{W}_{i} \leq 0, \quad j \in U_{u k}^{i}, j \neq i, \\
& \widetilde{P}_{j}-\widetilde{W}_{i} \geq 0, \quad j \in U_{u k^{\prime}}^{i} j=i,
\end{aligned}
$$

with

$$
\begin{aligned}
& \widetilde{\phi}_{i 11}=\widetilde{P}_{i} A_{i}+A_{i}^{T} \widetilde{P}_{i}+\sum_{j \in U_{k}^{i}} \lambda_{i j}\left(\widetilde{P}_{j}-W_{i}\right)+\widetilde{Q}_{1 i}+\widetilde{N}_{1} A_{i}+A_{i}^{T} \widetilde{N}_{1}^{T}+\tau_{1 i} \widetilde{R}_{1}, \\
& \widetilde{\phi}_{i 12}=-\widetilde{N}_{1}+A_{i}^{T} \widetilde{N}_{2}^{T}, \\
& \widetilde{\phi}_{i 13}=\widetilde{N}_{1} B_{i}+A_{i}^{T} \widetilde{N}_{3}^{T}+\widetilde{P}_{i} B_{i}, \\
& \widetilde{\phi}_{i 14}=\widetilde{N}_{1} C_{i}+A_{i}^{T} \widetilde{N}_{4}^{T}+\widetilde{P}_{i} C_{i}, \\
& \widetilde{\phi}_{i 22}=-\widetilde{N}_{2}-\widetilde{N}_{2}^{T}+\widetilde{Q}_{2 i}+\tau_{2 i} \widetilde{R}_{2} \text {, } \\
& \widetilde{\phi}_{i 23}=\widetilde{N}_{2} B_{i}-\widetilde{N}_{3}^{T}, \\
& \widetilde{\phi}_{i 24}=\widetilde{N}_{2} C_{i}-\widetilde{N}_{4}^{T}, \\
& \widetilde{\phi}_{i 33}=\widetilde{N}_{3} B_{i}+B_{i}^{T} \widetilde{N}_{3}^{T}-\widetilde{Q}_{1 i}+\sum_{j \in U_{k}^{i}} \lambda_{i j} \tau_{1 j} \widetilde{Q}_{1 i}-\lambda_{k}^{i} \tau_{1 j} \widetilde{Q}_{1 i} \\
& \widetilde{\phi}_{i 34}=\widetilde{N}_{3} C_{i}+B_{i}^{T} \widetilde{N}_{4}^{T}, \\
& \widetilde{\phi}_{i 44}=\widetilde{N}_{4} C_{i}+C_{i}^{T} \widetilde{N}_{4}^{T}-\widetilde{Q}_{2 i}+\sum_{j \in U_{k}^{i}} \lambda_{i j} \tau_{2 j} \widetilde{Q}_{2 i}-\lambda_{k}^{i} \tau_{2 j} \widetilde{Q}_{2 i}, \\
& \widetilde{\psi}_{i 33}=\widetilde{N}_{3} B_{i}+B_{i}^{T} \widetilde{N}_{3}^{T}-\widetilde{Q}_{1 i}+\sum_{j \in U_{k}^{i}} \lambda_{i j} \tau_{1 j} \widetilde{Q}_{1 i}+\lambda_{d}^{i} \tau_{1 i} \widetilde{Q}_{1 i}-\lambda_{d}^{i} \tau_{1 j} \widetilde{Q}_{1 i}-\lambda_{k}^{i} \tau_{1 j} \widetilde{Q}_{1 i}, \\
& \widetilde{\psi}_{i 44}=\widetilde{N}_{4} C_{i}+C_{i}^{T} \widetilde{N}_{4}^{T}-\widetilde{Q}_{2 i}+\sum_{j \in U_{k}^{i}} \lambda_{i j} \tau_{2 j} \widetilde{Q}_{2 i}+\lambda_{d}^{i} \tau_{2 i} \widetilde{Q}_{2 i}-\lambda_{d}^{i} \tau_{2 j} \widetilde{Q}_{2 i}-\lambda_{k}^{i} \tau_{2 j} \widetilde{Q}_{2 i},
\end{aligned}
$$




$$
\begin{array}{llll}
\tilde{\psi}_{i 11}=\tilde{\phi}_{i 11}, & \tilde{\psi}_{i 12}=\tilde{\phi}_{i 12}, & \tilde{\psi}_{i 13}=\tilde{\phi}_{i 13}, & \tilde{\varphi}_{i 14}=\tilde{\phi}_{i 14}, \\
\tilde{\psi}_{i 22}=\tilde{\phi}_{i 22}, & \tilde{\psi}_{i 23}=\tilde{\phi}_{i 23}, & \tilde{\psi}_{i 24}=\tilde{\phi}_{i 24}, & \tilde{\psi}_{i 34}=\tilde{\phi}_{i 34},
\end{array}
$$

and $\lambda_{d}^{i}$ is a given lower bound for the unknown diagonal element.

Proof. $\tilde{\phi}_{i}<0$ can be written as

$$
\tilde{\phi}_{i}=\phi_{i}+\mathbb{D}_{i}^{T} F_{i}(t) \mathbb{G}_{i}^{T}+\mathbb{G}_{i} F_{i}^{T}(t) \mathbb{D}_{i}<0,
$$

where

$$
\begin{gathered}
\mathbb{G}_{i}^{T}=\left(\begin{array}{cccc}
L_{i}^{T} P_{i}+L_{i}^{T} N_{1}^{T} & L_{i}^{T} N_{2}^{T} & L_{i}^{T} N_{3}^{T} & L_{i}^{T} N_{4}^{T}
\end{array}\right), \\
\mathbb{D}_{i}=\left(\begin{array}{llll}
H_{1 i} & 0 & H_{2 i} & 0
\end{array}\right),
\end{gathered}
$$

and $\phi_{i}$ are defined in Theorem 3.2. According to the approach in [40] with Lemma 2.4 in [41], there exists a scalar $\varepsilon$ such that (4.12) are equivalents to

$$
\tilde{\varphi}_{i}=\varepsilon \varphi_{i}+\varepsilon^{2} \mathbb{D}_{i}^{T} \mathbb{D}_{i}+\mathbb{G}_{i} \mathbb{G}_{i}^{T}<0
$$

Introducing new variables $\widetilde{P}_{i}=\varepsilon P_{i}, \widetilde{Q}_{1 i}=\varepsilon Q_{1 i}, \widetilde{Q}_{2 i}=\varepsilon Q_{2 i}, \widetilde{R}_{1}=\varepsilon R_{1}, \widetilde{R}_{2}=\varepsilon R_{2}$, $\widetilde{W}_{i}=\varepsilon W_{i}$, and $\widetilde{N}_{k}=\varepsilon N_{k}(k=1, \ldots, 4)$, and with Schur's complement [42], yields inequalities (4.3). Similarly, it concludes (4.4) with the same proof. On the other hand, with the same variables substitution, we note that pre- and postmultiplying, respectively, (3.16)-(3.21) by a scalar $\varepsilon$ yield (4.5)-(4.10), which completes this proof.

Remark 4.2. It should be mentioned that Theorem 4.1 is an extension of (2.1) to uncertain neutral markovian jump systems (4.1) with incomplete transition descriptions. In fact, this technology is frequently adopted in dealing with the robust stability analysis of uncertain systems.

\section{Examples}

In order to show the effectiveness of the approaches presented in the above sections, two numerical examples are provided.

Example 5.1. Consider the MJLS (2.1) with four operation modes whose state matrices are listed as follows:

$$
A_{1}=\left(\begin{array}{cc}
-1.15 & 0 \\
0 & -1.5
\end{array}\right), \quad A_{2}=\left(\begin{array}{cc}
-3.15 & 0 \\
0 & -7.1
\end{array}\right)
$$




$$
\begin{array}{rlr}
A_{3} & =\left(\begin{array}{cc}
-1.38 & 0 \\
0 & -1.8
\end{array}\right), & A_{4}=\left(\begin{array}{cc}
-2.95 & 0 \\
0 & -3.65
\end{array}\right), \\
B_{1}=\left(\begin{array}{cc}
0.02 & -0.02 \\
0.04 & -0.05
\end{array}\right), & B_{2}=\left(\begin{array}{cc}
-0.05 & -0.06 \\
0.07 & -0.07
\end{array}\right), \\
B_{3}=\left(\begin{array}{cc}
0.08 & -0.08 \\
-0.03 & 0.06
\end{array}\right), & B_{4}=\left(\begin{array}{cc}
0.07 & -0.05 \\
0.09 & 0.03
\end{array}\right), \\
C_{1}=\left(\begin{array}{cc}
0.29 & 0 \\
0 & 0.36
\end{array}\right), & C_{2}=\left(\begin{array}{cc}
0.35 & 0 \\
0 & -0.07
\end{array}\right), \\
C_{3}=\left(\begin{array}{cc}
0.03 & 0 \\
0 & 0.06
\end{array}\right), & C_{4}=\left(\begin{array}{cc}
0.28 & 0.01 \\
0.02 & 0.07
\end{array}\right) .
\end{array}
$$

The partly transition rate matrix $\Pi$ is considered as

$$
\text { Case I }: \Pi=\left[\begin{array}{cccc}
-1.3 & 0.2 & ? & ? \\
? & ? & 0.3 & 0.3 \\
0.6 & ? & -1.5 & ? \\
0.4 & ? & ? & ?
\end{array}\right], \quad \text { Case II }: \Pi=\left[\begin{array}{cccc}
-1.3 & 0.2 & 0.6 & 0.5 \\
0.2 & -0.8 & 0.3 & 0.3 \\
0.6 & 0.7 & -1.5 & 0.2 \\
0.4 & 0.1 & 0.4 & -0.9
\end{array}\right]
$$

Our purpose here is to check the stability of the above system for the two different cases of transition probabilities. For Case I, it is clear to see that $\lambda_{22}$ and $\lambda_{44}$ are not valued, one can set $\lambda_{d}^{2}=-0.8, \lambda_{d}^{2}=-0.9$, and let $\tau_{12}=1.1000, \tau_{13}=0.8000, \tau_{14}=0.9900, \tau_{21}=$ $\tau_{11}, \tau_{22}=\tau_{12}, \tau_{23}=\tau_{13}$, and $\tau_{24}=\tau_{14}$. Solving the inequalities in Theorem 3.2 using LMI toolbox, the maximum of the time delay $\tau_{11}$ can be computed as $\tau_{11}=1.1210$. However, in Case II, the maximum of the time delay $\tau_{11}$ can be computed as $\tau_{11}=2.0530$ by Theorem 3.1. It is easily seen that the more transition probabilities knowledge we have, the larger the maximum of delay can be obtained for ensuring stability. This shows the trade-off between the cost of obtaining transition probabilities and the system performance.

Furthermore, when the transition probabilities are not fully known, as the delay for one of the subsystems decreases, the maximum of other delays may increase. However, when all transition probabilities are fully known, the conclusion may be on the opposite in some interval. In fact, the above observation is in accordance with the actual. Then, we assume that $\lambda_{d}^{2}=-0.8, \lambda_{d}^{2}=-0.9$, and let $\tau_{12}=1.1000, \tau_{14}=0.9900, \tau_{21}=\tau_{11}, \tau_{22}=\tau_{12}, \tau_{23}=\tau_{13}$, and $\tau_{24}=\tau_{14}, \tau_{13}$ be different with $\tau_{11}$, and with the same computation in Theorem 3.1, as shown in Table 1. However, just according to the approach of Theorem 3 in [32], not introducing some other free matrices and some other skills, we cannot find the feasible solutions which contain time delay to verify the stability of the system. Therefore, this example shows that the stability criterion in this paper gives much less conservative delay-dependent stability conditions. This example also shows that the approach presented in this paper is effectiveness. 
Table 1: The maximal allowable delays $\tau_{11}$ of Example 5.1 for different values of $\tau_{12}$ in a different case.

\begin{tabular}{lcccccccccc}
\hline Case & \multicolumn{1}{c}{ I } & \multicolumn{1}{c}{ II } \\
\hline$\tau_{13}$ & 0.80 & 0.75 & 0.70 & 0.65 & 0.60 & 0.75 & 0.80 & 0.85 & 0.90 & 0.95 \\
$\tau_{11}$ & 1.1210 & 1.1960 & 1.2710 & 1.3460 & 1.4210 & 1.9280 & 2.0530 & 2.1780 & 2.3030 & 2.4279 \\
\hline
\end{tabular}

Example 5.2. Consider the above MJLS in Example 5.1 with partially unknown transition probabilities of Case I, and the uncertain structure matrices are described by (4.2) where

$$
\begin{gathered}
L_{1}=\left(\begin{array}{c}
0.1 \\
0.2
\end{array}\right), \quad L_{2}=\left(\begin{array}{c}
0.2 \\
-0.4
\end{array}\right), \quad L_{3}=\left(\begin{array}{l}
0.4 \\
0.3
\end{array}\right), \quad L_{4}=\left(\begin{array}{l}
0.25 \\
0.27
\end{array}\right), \\
H_{11}=\left(\begin{array}{ll}
0.72 & 1.14
\end{array}\right), \quad H_{12}=\left(\begin{array}{lll}
1.22 & 1.66
\end{array}\right), \quad H_{13}=\left(\begin{array}{lll}
1.41 & -1.13
\end{array}\right), \quad H_{14}=\left(\begin{array}{ll}
-1.39 & 1.63
\end{array}\right), \\
H_{21}=\left(\begin{array}{ll}
-1.11 & 1.32
\end{array}\right), \quad H_{22}=\left(\begin{array}{lll}
1.31 & 1.14
\end{array}\right), \quad H_{23}=\left(\begin{array}{lll}
1.27 & 1.15
\end{array}\right), \quad H_{24}=\left(\begin{array}{ll}
-1.26 & 1.37
\end{array}\right) .
\end{gathered}
$$

In this case, we check the robust stability result provided by Theorem 4.1. One can also set $\lambda_{d}^{2}=-0.8, \lambda_{d}^{2}=-0.9$, and let $\tau_{12}=1.1000, \tau_{13}=0.7500, \tau_{14}=0.9900, \tau_{21}=\tau_{11}, \tau_{22}=\tau_{12}, \tau_{23}=\tau_{13}$, and $\tau_{24}=\tau_{14}$. Solving the inequalities in Theorem 4.1 by using LMI toolbox, the maximum of the time delay $\tau_{11}$ can be computed as $\tau_{11}=1.0892$. Some of the feasible solutions can be obtained as follows:

$$
\begin{gathered}
P_{1}=\left(\begin{array}{cc}
6.6762 & -1.8338 \\
-1.8338 & 9.5418
\end{array}\right), \quad P_{2}=\left(\begin{array}{cc}
6.6930 & -1.8283 \\
-1.8283 & 9.5446
\end{array}\right), \\
P_{3}=\left(\begin{array}{cc}
10.7916 & -5.0166 \\
-5.0166 & 12.0252
\end{array}\right), \quad P_{4}=\left(\begin{array}{cc}
10.7923 & -5.0178 \\
-5.0178 & 12.0274
\end{array}\right), \\
W_{1}=\left(\begin{array}{cc}
10.7928 & -270.5249 \\
260.4880 & 12.0285
\end{array}\right), \quad W_{2}=\left(\begin{array}{cc}
6.6915 & 371.7539 \\
-375.4115 & 9.5439
\end{array}\right), \\
W_{3}=\left(\begin{array}{cc}
10.7943 & 82.9434 \\
-92.9861 & 12.0338
\end{array}\right), \quad W_{4}=\left(\begin{array}{cc}
10.7920 & -212.1720 \\
202.1374 & 12.0265
\end{array}\right), \\
N_{4}=\left(\begin{array}{cc}
-0.3069 & 0.1342 \\
0.0548 & 0.1402
\end{array}\right) .
\end{gathered}
$$

In a word, this example shows that the robust stability condition of Theorem 4.1 is feasible. It is also approved that the approach provided in this paper is effectiveness.

Example 5.3. Consider the MJLS (2.1) with two operation modes whose state matrices are listed as follows:

$$
\begin{array}{ll}
A_{1}=\left(\begin{array}{cc}
-2.16 & 0.05 \\
-0.15 & -1.35
\end{array}\right), \quad A_{2}=\left(\begin{array}{cc}
-3.13 & -0.19 \\
0.21 & -2.21
\end{array}\right), \quad B_{1}=\left(\begin{array}{cc}
0.22 & -0.02 \\
0.54 & -0.05
\end{array}\right), \\
B_{2}=\left(\begin{array}{cc}
0.43 & -0.16 \\
0.27 & 1.07
\end{array}\right), \quad C_{1}=\left(\begin{array}{cc}
-0.08 & -0.06 \\
0.03 & 0.04
\end{array}\right), \quad C_{2}=\left(\begin{array}{cc}
0.05 & 0.31 \\
0.23 & -0.06
\end{array}\right) .
\end{array}
$$


The partly transition rate matrix $\Pi$ is considered as

$$
\Pi=\left[\begin{array}{ll}
? & ? \\
? & ?
\end{array}\right]
$$

The above matrix (5.8) implies that systems (2.1) with all transition probabilities are not known, which viewed the systems as switched systems with arbitrary switching. Given that $\tau_{11}=2.4279, \tau_{12}=1.1000, \tau_{13}=0.95, \tau_{14}=0.9900, \tau_{21}=\tau_{11}, \tau_{22}=\tau_{12}, \tau_{23}=\tau_{13}$, and $\tau_{24}=\tau_{14}$, according to the approach of Theorem 3.2, we cannot find the feasible solutions. However, using the Matlab LMI toolbox, we solve the LMI in Corollary 3.5, and the feasible solutions can be obtained as follows:

$$
\begin{gathered}
P=\left(\begin{array}{cc}
15.9364 & 0.7491 \\
0.7491 & 11.2785
\end{array}\right), \quad R_{1}=\left(\begin{array}{ll}
2.7619 & 0.2382 \\
0.2382 & 1.5829
\end{array}\right), \\
R_{2}=\left(\begin{array}{ll}
0.9940 & 0.0974 \\
0.0974 & 0.5010
\end{array}\right), \quad Q_{1}=\left(\begin{array}{ll}
6.8645 & 0.3173 \\
0.3173 & 7.3064
\end{array}\right), \quad Q_{2}=\left(\begin{array}{ll}
4.3109 & 0.1752 \\
0.1752 & 3.8836
\end{array}\right) .
\end{gathered}
$$

For the case of all transition probabilities that are unknown, this example also shows that Corollary 3.5 is less conservative than Theorem 3.2 on the stability analysis for the neutral markovian jump system.

\section{Conclusion}

The delay-dependent stability for neutral markovian jump systems with partly known transition probabilities has been investigated. Based on a new class of stochastic LyapunovKrasovskii functionals constructed, and combined with the technique of analysis for matrix inequalities, some new stability criteria are obtained. The main contribution of this paper contains the following two-fold: one is the extension of delay-dependent stability conditions for markovian jump delay systems to markovian neutral jump systems; the other is the new method presented to decrease the conservative brought by the markovian jump with partly known transition probabilities. The future work is to investigate the systems with modedependent interval mixed time delays and the systems with unsynchronised control input. Three examples have shown the effectiveness of the conditions presented in this paper.

\section{Nomenclature}

$R^{n}: \quad n$-dimensional real space

$R^{m \times n}$ : Set of all real $m$ by $n$ matrices

$x^{T}$ or $A^{T}$ : Transpose of vector $x$ (or matrix $A$ )

$P>0: \quad(P<0$, resp. $)$ Matrix $P$ is symmetric positive (negative, resp.) definite

$P \geq 0: \quad(P \leq 0$, resp.) Matrix $P$ is symmetric positive (negative, resp.) semidefinite

$\star: \quad$ The elements below the main diagonal of a symmetric block matrix

$x_{t}(\theta): \quad x(t+\theta), \theta \in[-\tau, 0]$. 


\section{Acknowledgments}

This work was supported by the Mathematical Tianyuan Foundation of China (Grant no. 11126305), the Natural Science Foundation of Yunnan Province (Grant no. 2011FZ172), the Natural Science Foundation of Yunnan Province (Grant no. 2011FZ169), the Natural Science Foundation of Yunnan Province (Grant no. 2009CD019), and the Youth Foundation of Yunnan University of Nationalities (Grant no. 11QN07).

\section{References}

[1] D. Liberzon and A. S. Morse, "Basic problems in stability and design of switched systems," IEEE Control Systems Magazine, vol. 19, no. 5, pp. 59-70, 1999.

[2] G. Zhai, X. Xu, H. Lin, and A. N. Michel, "Analysis and design of switched normal systems," Nonlinear Analysis. Theory, Methods E Applications A, vol. 65, no. 12, pp. 2248-2259, 2006.

[3] R. DeCarlo, M. S. Branicky, S. Pettersson, and B. Lennartson, "Perspectives and results on the stability and stabilizability of hybrid systems," Proceedings of the IEEE, vol. 88, no. 7, pp. 1069-1082, 2000.

[4] Z. Sun and S. S. Ge, "Analysis and synthesis of switched linear control systems," Automatica, vol. 41, no. 2, pp. 181-195, 2005.

[5] Z.-H. Guan and H. Zhang, "Stabilization of complex network with hybrid impulsive and switching control," Chaos, Solitons \& Fractals, vol. 37, no. 5, pp. 1372-1382, 2008.

[6] I. Cervantes, R. Femat, and J. -L. Ramos, "Study of a class of hybrid-time systems," Chaos, Soliton $\mathcal{E}$ Fractals, vol. 32, no. 3, pp. 1081-1095, 2007.

[7] X. Liu, K.-L. Teo, H. Zhang, and G. Chen, "Switching control of linear systems for generating chaos," Chaos, Solitons \& Fractals, vol. 30, no. 3, pp. 725-733, 2006.

[8] X. M. Sun, J. Fu, H. F. Sun, and J. Zhao, "Stability of linear switched neutral delay systems," Proceedings of the Chinese Society for Electrical Engineering, vol. 25, no. 23, pp. 42-46, 2005.

[9] X. Liu and S. Yuan, "On designing $H_{\infty}$ fault estimator for switched nonlinear systems of neutral type," Communications in Nonlinear Science and Numerical Simulation, vol. 16, no. 11, pp. 4379-4389, 2011.

[10] D. Liu, X. Liu, and S. Zhong, "Delay-dependent robust stability and control synthesis for uncertain switched neutral systems with mixed delays," Applied Mathematics and Computation, vol. 202, no. 2, pp. 828-839, 2008.

[11] L. Xiong, S. Zhong, M. Ye, and S. Wu, "New stability and stabilization for switched neutral control systems," Chaos, Solitons \& Fractals, vol. 42, no. 3, pp. 1800-1811, 2009.

[12] L.-L. Xiong, S.-M. Zhong, and M. Ye, "Delay-dependent BIBO stability analysis of switched uncertain neutral systems," Mathematical and Computer Modelling, vol. 53, no. 9-10, pp. 1607-1620, 2011.

[13] C.-H. Lien and K.-W. Yu, "Stability criteria for uncertain neutral systems with interval time-varying delays," Chaos, Solitons \& Fractals, vol. 38, no. 3, pp. 650-657, 2008.

[14] C.-H. Lien, K.-W. Yu, Y.-J. Chung, Y.-F. Lin, L.-Y. Chung, and J.-D. Chen, "Exponential stability analysis for uncertain switched neutral systems with interval-time-varying state delay," Nonlinear Analysis. Hybrid Systems, vol. 3, no. 3, pp. 334-342, 2009.

[15] K.-W. Yu, "Switching signal design for global exponential stability of uncertain switched neutral systems," Mathematical Problems in Engineering, vol. 2009, Article ID 191760, 17 pages, 2009.

[16] T. F. Li, G. M. Dimirovski, Y. Y. Liu, and J. Zhao, "Improved stability of a class of switched neutral systems vi-a Lyapunov-Krasovskii functionals and an average dwell-time scheme," International Journal of Systems Science, vol. 53, no. 1, pp. 1-13, 2012.

[17] Z. Xiang, Y.-N. Sun, and Q. Chen, "Robust reliable stabilization of uncertain switched neutral systems with delayed switching," Applied Mathematics and Computation, vol. 217, no. 23, pp. 9835-9844, 2011.

[18] D. Zhang and L. Yu, "Exponential stability analysis for neutral switched systems with interval timevarying mixed delays and nonlinear perturbations," Nonlinear Analysis. Hybrid Systems, vol. 6, no. 2, pp. 775-786, 2012.

[19] P. Balasubramaniam, A. Manivannan, and R. Rakkiyappan, "Exponential stability results for uncertain neutral systems with interval time-varying delays and Markovian jumping parameters," Applied Mathematics and Computation, vol. 216, no. 11, pp. 3396-3407, 2010.

[20] Y. Zhang, X. Liu, H. Zhu, and S. Zhong, "Stability analysis and control synthesis for a class of switched neutral systems," Applied Mathematics and Computation, vol. 190, no. 2, pp. 1258-1266, 2007. 
[21] D. Du, B. Jiang, P. Shi, and S. Zhou, "Robust $l_{2}-l_{\infty}$ control for uncertain discrete-time switched systems with delays," Circuits, Systems, and Signal Processing, vol. 25, no. 6, pp. 729-744, 2006.

[22] T.-F. Li, J. Zhao, and G. M. Dimirovski, "Stability and $L_{2}$-gain analysis for switched neutral systems with mixed time-varying delays," Journal of the Franklin Institute, vol. 348, no. 9, pp. 2237-2256, 2011.

[23] E.-K. Boukas, Stochastic Switching Systems: Analysis and Design, Birkhäuser, Berlin, Germany, 2005.

[24] H. Lin and P. J. Antsaklis, "Stability and stabilizability of switched linear systems: a survey of recent results," IEEE Transactions on Automatic Control, vol. 54, no. 2, pp. 308-322, 2009.

[25] D. Liberzon, Switching in Systems and Control, Birkhäuser, Berlin, Germany, 2003.

[26] L. Xiong, J. Tian, and X. Liu, "Stability analysis for neutral Markovian jump systems with partially unknown transition probabilities," Journal of the Franklin Institute, vol. 349, no. 6, pp. 2193-2214, 2012.

[27] P. Balasubramaniam, R. Krishnasamy, and R. Rakkiyappan, “Delay-dependent stability criterion for a class of non-linear singular Markovian jump systems with mode-dependent interval time-varying delays," Communications in Nonlinear Science and Numerical Simulation, vol. 17, no. 9, pp. 3612-3627, 2012.

[28] P. Balasubramaniam, S. Lakshmanan, and A. Manivannan, "Robust stability analysis for Markovian jumping interval neural networks with discrete and distributed time-varying delays," Chaos, Solitons E Fractals, vol. 45, no. 4, pp. 483-495, 2012.

[29] P. Balasubramaniam, R. Krishnasamy, and R. Rakkiyappan, "Delay-interval-dependent robust stability results for uncertain stochastic systems with Markovian jumping parameters," Nonlinear Analysis. Hybrid Systems, vol. 5, no. 4, pp. 681-691, 2011.

[30] L. Zhang and E.-K. Boukas, "Stability and stabilization of Markovian jump linear systems with partly unknown transition probabilities," Automatica, vol. 45, no. 2, pp. 463-468, 2009.

[31] Q. Ma, S. Y. Xu, and Y. Zou, "Stability and synchronization for Markovian jump neural networks with partly unknown transition probabilities," Neurocomputing, vol. 74, no. 17, pp. 3404-3411, 2011.

[32] Y. Zhang, Y. He, M. Wu, and J. Zhang, "Stabilization for Markovian jump systems with partial information on transition probability based on free-connection weighting matrices," Automatica, vol. 47, no. 1, pp. 79-84, 2011.

[33] L. Zhang and J. Lam, "Necessary and sufficient conditions for analysis and synthesis of Markov jump linear systems with incomplete transition descriptions," IEEE Transactions on Automatic Control, vol. 55, no. 7, pp. 1695-1701, 2010.

[34] L. Zhang, E.-K. Boukas, and J. Lam, "Analysis and synthesis of Markov jump linear systems with time-varying delays and partially known transition probabilities," IEEE Transactions on Automatic Control, vol. 53, no. 10, pp. 2458-2464, 2008.

[35] L. X. Zhang, E.-K. Boukas, and L. Baron, "Fault detection for discrete-time Markov jump linear systems with partially known transition probabilities," in Proceedings of the 47th IEEE Conference on Decision and Control, pp. 1054-11059, Cancun, Mexico, December 2008.

[36] L. Zhang and E.-K. Boukas, "Mode-dependent $H_{\infty}$ filtering for discrete-time Markovian jump linear systems with partly unknown transition probabilities," Automatica, vol. 45, no. 6, pp. 1462-1467, 2009.

[37] X. Luan, F. Liu, and P. Shi, "Finite-time filtering for non-linear stochastic systems with partially known transition jump rates," IET Control Theory \& Applications, vol. 4, no. 5, pp. 735-745, 2010.

[38] S. Xu, J. Lam, and X. Mao, "Delay-dependent $H_{\infty}$ control and filtering for uncertain Markovian jump systems with time-varying delays," IEEE Transactions on Circuits and Systems I, vol. 54, no. 9, pp. 20702077, 2007.

[39] A. V. Skorokhod, Asymptotic Methods in the Theory of Stochastic Differential Equations, American Mathematical Society, Providence, RI, USA, 1989.

[40] Q.-L. Han, “A descriptor system approach to robust stability of uncertain neutral systems with discrete and distributed delays," Automatica, vol. 40, no. 10, pp. 1791-1796, 2004.

[41] L. Xie, "Output feedback $H_{\infty}$ control of systems with parameter uncertainty," International Journal of Control, vol. 63, no. 4, pp. 741-750, 1996.

[42] S. Boyd, L. El Ghaoui, E. Feron, and V. Balakrishnan, Linear Matrix Inequalities in System and Control Theory, Society for Industrial and Applied Mathematics, Philadelphia, Pa, USA, 1994. 


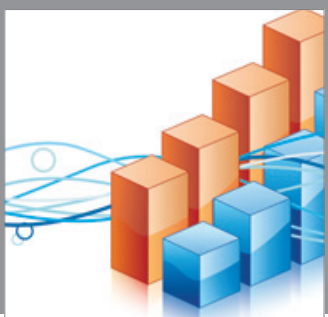

Advances in

Operations Research

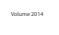

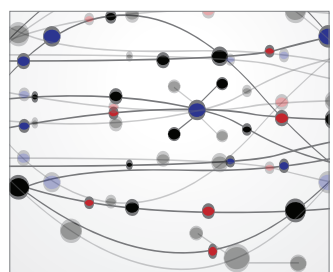

\section{The Scientific} World Journal
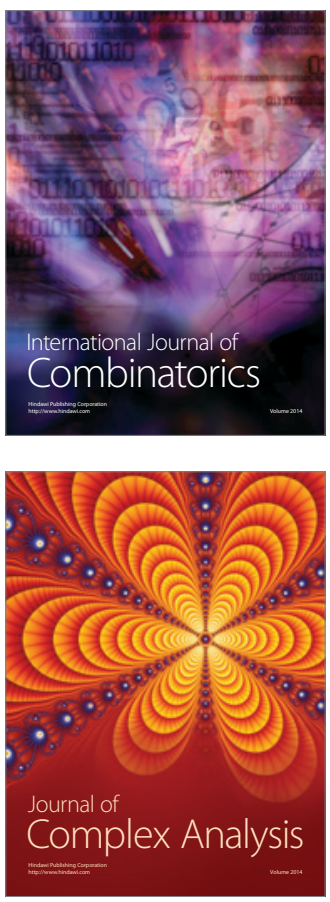

International Journal of

Mathematics and

Mathematical

Sciences
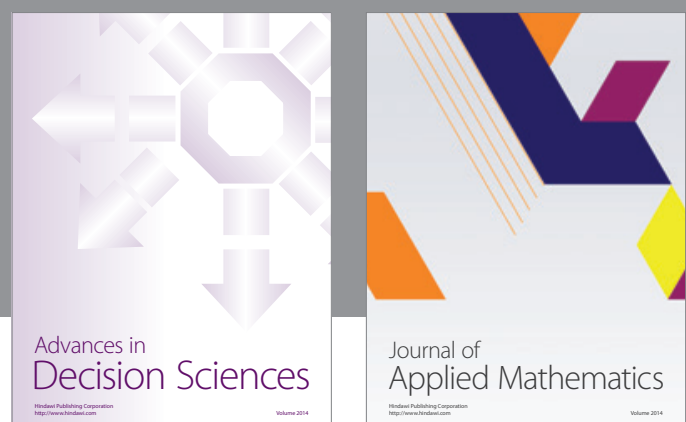

Journal of

Applied Mathematics
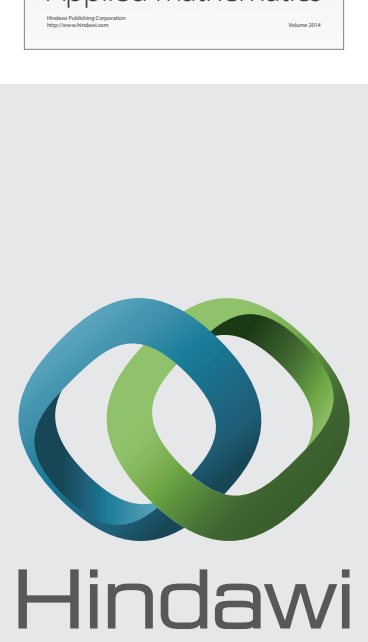

Submit your manuscripts at http://www.hindawi.com
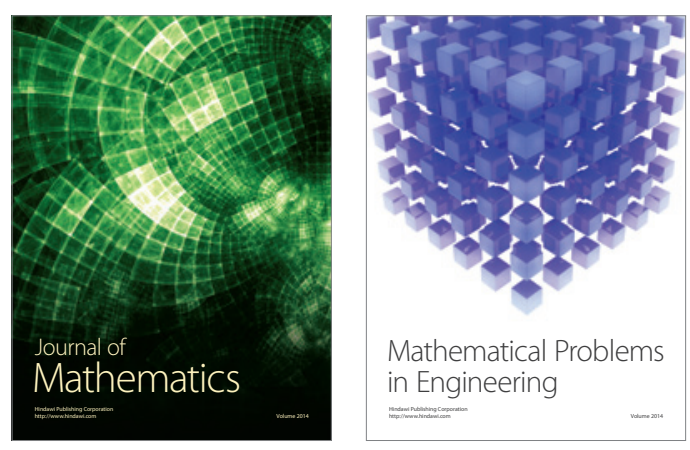

Mathematical Problems in Engineering
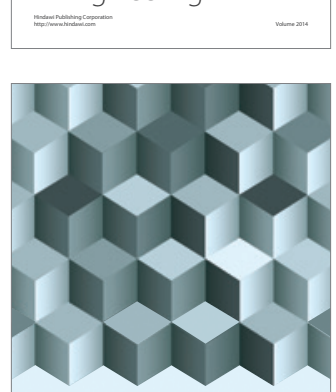

Journal of

Function Spaces
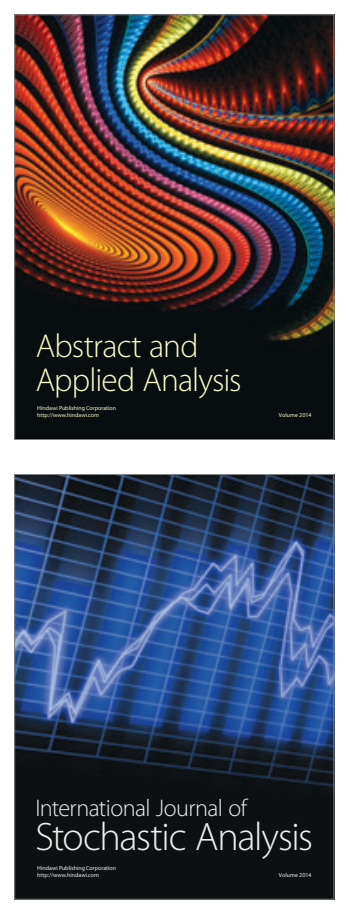

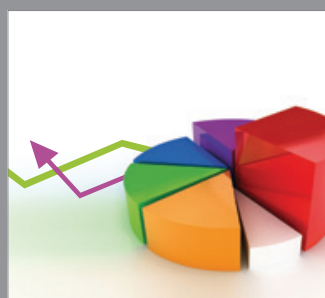

ournal of

Probability and Statistics

Promensencen
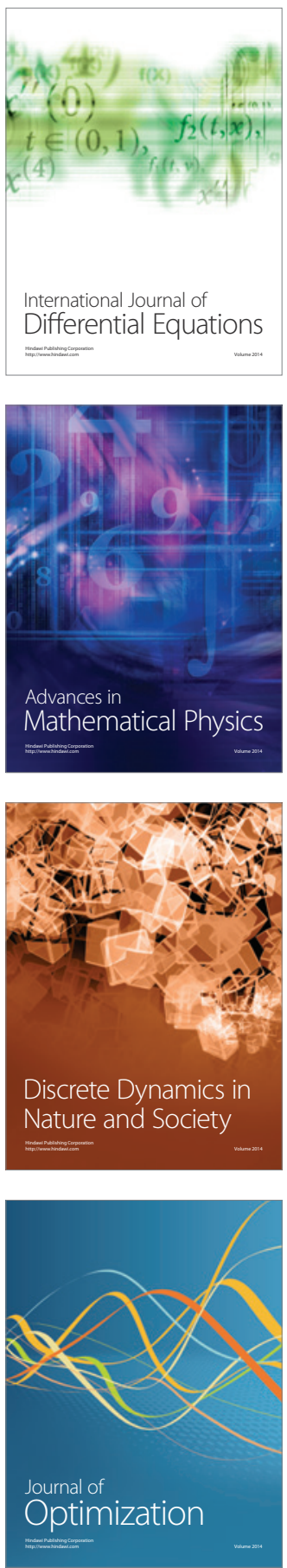